\title{
MORFOLOGIA DOS MINERAIS DE OURO E ARSENOPIRITA NO DEPÓSITO MARIA LÁZARA (GUARINOS, GOIÁS) E SUAS IMPLICAÇÕES NOS MECANISMOS DE DEPOSIÇÃO
}

\author{
GÊNOVA M. PULZ*, ÉDER S. MARTINS* e REINHARDT A. FUCK*
}

\begin{abstract}
MORPHOLOGY OF GOLD MINERALS AND ARSENOPYRITE IN THE MARIA LÁZARA DEPOSIT (GUARINOS, GOIÁS) AND ITS IMPLICATION IN THE DEPOSITION MECHANISMS. Morphology of gold and arsenopyrite grains occurring in the hydrothermal alteration halo of the Maria Lázara deposit (Goiás) was studied using optical and scanning electron microscopy. Arsenopyrite displays deformational and recovery features associated with corrosion textures (dissolution microcavities) due to late stage percolation fluids. The gold minerals investigated show a wide variety of forms which indicate the precipitation mechanism that operated after intense metabasalt sulphidation. Textural and chemical relationships of the gold-arsenopyrite association demonstrate that gold deposition in the veins and the hydrothermal halo was independent from the deformation and metamorphism mechanisms of arsenopyrite and hydrotermalized matrix. Gold precipitation from hydrothermal fluid after the arsenopyrite deposition can be related with: 1. oxidation of hydrothermal fluid and, 2. electro-chemical reactions due to high conductivity of the sulphide surfaces. Gold minerals associated with the latest deposition stage exhibit abundant features on their surface, suggesting post-depositional changes in physico-chemical conditions of the hydrothermal fluid. These changes during the evolution of the hydrothermal system at the Maria Lázara gold deposit lead to remobilization processes resulting in the erratic distribution of gold grade within the ore zone.
\end{abstract}

Keywords: Gold minerals, arsenopyrite, morphology, hydrothermal system, depositional mechanisms, electro-chemical reactions. RESUMO A morfologia dos grãos de ouro e de arsenopirita que ocorrem nos veios e no halo de alteração
hidrotermal do depósito Maria Lázara (Goiás) foi estudada sob microscópio óptico com luz refletida e com microscópio
eletrônico de varredura (MEV). A arsenopirita revelou feições de deformação, de recuperação e texturas de corrosão
(microcavidades de dissolução), as quais indicam a percolação de fluidos tardios. As espécies auríferas analisadas
mostraram uma ampla variedade de formas que fornecem evidências dos mecanismos de sua precipitação após a intensa
sulfetação do metabasalto. As relações texturais e químicas da associação ouro-arsenopirita demonstram que a
deposição do ouro, tanto nos veios como no halo hidrotermal, independe dos processos de deformação e recristalização
metamórfica da arsenopirita ou da matriz hidrotermalizada. A precipitação do ouro livre diretamente do fluido
hidrotermal após a deposição da arsenopirita pode estar relacionada com: 1. a oxidação do fluido hidrotermal e, 2 .
reações eletro-químicas promovidas pela elevada conduti vidade elétrica dos sulfetos preexistentes. Marcas na superficie
dos minerais auríferos do estágio tardio de deposição sugerem mudanças pós-deposicionais nas condições físico-
químicas do fluido hidrotermal. Essas mudanças durante a evolução do sistema hidrotermal no depósito Maria Lázara
favoreceram processos de remobilização dos minerais auriferos, contribuindo para a distribuição errática dos teores de
ouro no nível mineralizado.

Palavras-chaves: Minerais auríferos, arsenopirita, morfologia, sistema hidrotermal, mecanismos de deposição, reações eletroquímicas.

INTRODUÇÃO A freqüência com que o ouro é encontrado junto a sulfetos é de interesse tanto prospective como metalúrgico. Neste sentido, a arsenopirita pode conter mais de $1,6 \%$ de Au em peso (Picot \& Marcoux 1987) sob a forma de finas partículas coloidais, inferiores a $0,1 \mu \mathrm{m}$ (Bakken et al. 1989), ou em solução sólida relacionada com a proporção de As (Cabri et al. 1989, Johan et al. 1989). A liberação do ouro submicroscópico durante estágios de microfraturamento e recristalização dos sulfetos é um dos modelos sugeridos para justificar a constante associação do ouro livre com a arsenopirita e pirita ricas em As. Por conseqüência, o entendimento dos mecanismos de deformação e metamorfismo dos sulfetos tornou-se um pré-requisito metalogenético, pois fornece dados acerca dos fenômenos de remobilização do minério hipogênico e seus efeitos na diluição e concentração dos metais (Vokes 1969, Nebel et al. 1991).

Nas zonas de cisalhamento que afetam os terrenos de greenstone belts da região de Crixás (Goiás) há muitas ocorrências auríferas, dentre as quais destaca-se o Depósito Maria Lázara. Neste depósito, o ouro mostra-se acompanhado por arsenopirita, cujas relações texturais fornecem subsídios para a discussão dos processos de deformação dos sulfetos e sua relação com os mecanismos de deposição e remobilização do metal precioso durante a percolação de fluidos hidrotermais.

\section{GEOLOGIA DO DEPÓSITO MARIA LÁZARA}

$\mathrm{O}$

Depósito Maria Lázara está situado cerca de $8 \mathrm{~km}$ a sul da cidade de Guarinos, na porção central do estado de Goiás (Fig. 1). Insere-se no contexto da zona de cisalhamento Carroça (Pulz 1990, Pulz et al. 1991a), que afetou a principal unidade estratigráfica do greenstone belt Guarinos, constituída por metabasaltos com intercalações de sedimentos carbonosos e formações ferríferas (Danni et al 1986).

O nível mineralizado contém ouro disseminado em um amplo halo de alteração hidrotermal no interior do qual alojase um reduzido sistema de veios. A fase hidrotermal envolveu a participação de $\mathrm{CO}_{2}, \mathrm{~K}_{2} \mathrm{O}$ e As, como revela o alinhamento de carbonates na foliação milonítica, a neo-formação de biotita em junções tríplices e de porfíroblastos de arsenopirita em xistos. $\mathrm{O}$ crescimento sintectônico dos fenoblastos de arsenopirita é sugerido pela presença de raros augen elipsoidais paralelos à foliação milonítica. Entretanto, a arsenopirita é mais freqüente sob a forma de fenoblastos idiomórficos, isentos de deformação, sugerindo que os processos de sulfetação do metabasalto prosseguiram após o pico da deformação.

A íntima relação entre a percolação episódica de fluidos hidrotermais (Pulz et al. 1992a) e o cisalhamento está registrada nas feições texturais exibidas pela mineralogia hidrotermal. Estas incluem a extinção ondulante do quartzo, sigmóides 


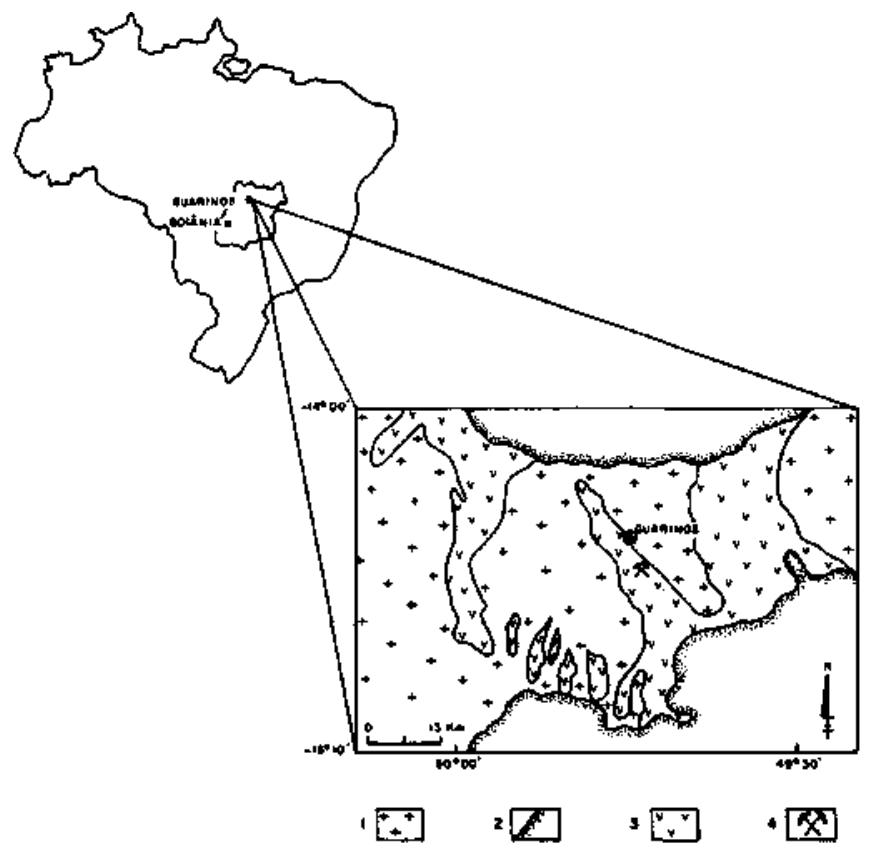

Figura l-Esboço geológico da região de Guarinos, destacando a localização do Depósito Maria Lázara

Figure 1 - Geological outline of the Guarinos area with the localization of the Maria Lázara gold deposit. 1. Granite-gnaisses blocks; 2. Proterozoic cover; 3. Greenstone Belts; 4. Maria Lázara deposit

intragranulares na biotita, junto a sericita e clorita com textura beard e estilolitos em veios de quartzo-carbonato.

Os minerais opacos que acompanham o ouro são abundantes e diversificados, incluindo a arsenopirita, pirita, calcopirita, pirrotita, galena, esfalerita, molibdenita, prata nativa, bismuto nativo, bismutinita, csiklovaíta $\left(\mathrm{Bi}_{2} \mathrm{TeS}_{2}\right)$ e joseíta-B $\left(\mathrm{Bi}_{4} \mathrm{Te}_{2} \mathrm{~S}\right)$. Raras inclusões de monazita foram observadas na arsenopirita (Pulz et al 1992b). Recentes estudos mineralógicos identificaram scheelita na associação (Michel et al 1992).

PROCEDIMENTO ANALÍTICO Estudos minerográficos, utilizando microscópio petrográfico com luz refletida foram conduzidos em 80 seções polidas obtidas de amostras coletadas até $25 \mathrm{~m}$ de profundidade no halo de alteração hidrotermal e em veios de quartzo e de quartzo-carbonato. Os dados foram complementados com a obtenção de imagens tridimensionais qualitativas, utilizando-se microscópio eletrônico de varredura (MEV-modelo Jeol JSM-840A), operando sob a forma topográfica e de elétrons secundários e retroespalhados, sob uma voltagem $10^{-9} \mathrm{kV}$ e corrente de $8 \AA$, no Instituto de Ciências Biológicas da Universidade de Brasília.

Amostras de veios de quartzo-carbonato foram selecionadas para análise da morfologia dos grãos de ouro e de suas relações texturais com a arsenopirita e com a matriz. As amostras foram divididas em dois grupos: 1. as amostras contendo ouro e arsenopirita visíveis foram reduzidas com o emprego de alicate a fragmentos de tamanho compatível com o suporte usado no MEV; 2. as amostras contendo ouro intersticial entre os grãos de quartzo e carbonato foram submetidas a reações de ataque com $\mathrm{HF}+\mathrm{HNO}_{3}$ a quente por duas horas. $\mathrm{O}$ resíduo foi filtrado com água destilada e analisado sob o MEV.

RELAÇÕES TEXTURAIS DA ASSOCIAÇÃO OUROARSENOPIRITA No depósito Maria Lázara, a arsenopirita é a espécie de sulfeto dominante. Mostra uma diversidade de texturas (cristais de tamanho variável, fraturas, subgrãos e extinção ondulante) que revelam o tipo e os mecanismos de sua deformação. $\mathrm{O}$ fraturamento dos fenoblastos constitui a principal evidência de sua deformação. Em uma mesma seção polida ocorrem grãos de arsenopirita intensamente fraturados adjacentes a grãos sem fraturas. A análise da distribuição de freqüência das fraturas em seções laterais de arsenopirita indica que as mesmas independem do tamanho do grão (Fig. 2). Ao microscópio, a arsenopirita mostra extinção ondulante e subgrãos, cuja densidade, distribuição e tamanho variam no mesmo grão e entre grãos distintos. Em conjunto, essas relações texturais denunciam a deformação da estrutura cristalina de alguns grãos de arsenopirita no nível mineralizado durante o cisalhamento.

Feições de corrosão são freqüentes no núcleo dos cristais de arsenopirita, como indicam as abundantes microcavidades de dissolução, independente das texturas de deformação. Os poros de dissolução apresentam formas irregulares, em geral de contorno arredondado e dimensões inferiores a $10 \mu \mathrm{m}$ (Fig. 3).

Os minerais aurfferos consistem de maldpnita $\left(\mathrm{Au}_{2} \mathrm{Bi}\right)$, ouro nativo e intercrescimentos de ouro nativo com fases do sistema Bi-Te-S. Ocorrem tanto em contato direto com a arsenopirita como sobre os planos de clivagem da biotita e

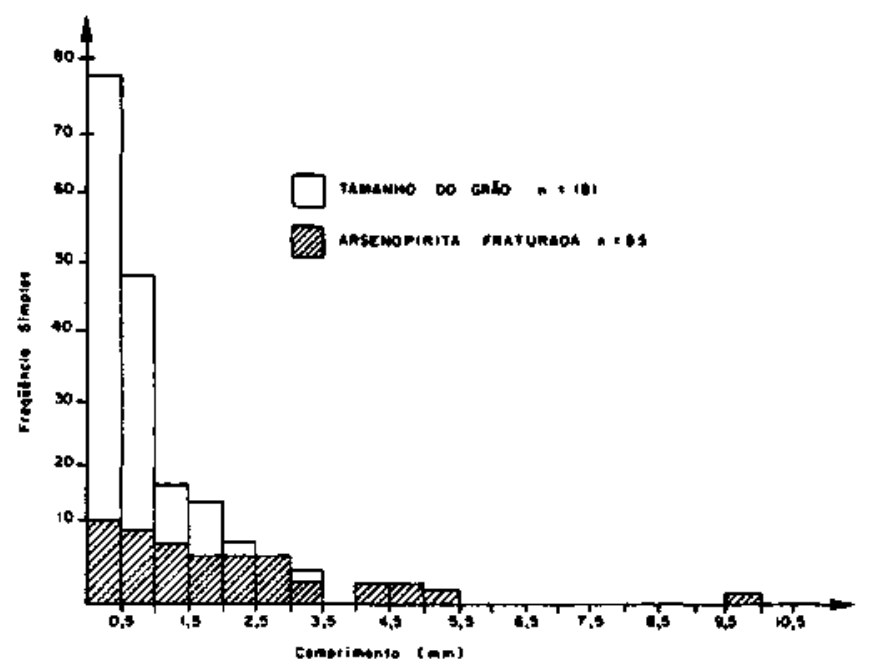

Figura 2 - Análise estatística das características físicas dos cristais de arsenopirita do Depósito Maria Lázara. Diagrama de freqüencia simples, ilustrando a variação do comprimento dos cristais de arsenopirita, no depósito Maria Lázara, e a freqüência de suas fraturas nas distintas classes granulométricas (n-número total de medidas)

Figure 2 - Statistical analysis of the physics characteristics of the arsenopyrite crystals from the Maria Lázara deposit. Simple frequency diagram showing length variation of arsenopyrite crystals, as well as fracture frequency in the different granulometric classes ( $n=$ numbers of total measurements)

clorita hidrotermais ou de forma intersticial entre grãos de quartzo e carbonato. As relações texturais exibidas pela associação ouro-arsenopirita são caracterizadas por contatos planares e abruptos. As observações com auxilio de microscópio eletrônico de varredura revelaram espécies auríferas de tamanho e forma variáveis, sobre as faces, fraturas e planos de clivagem da arsenopirita. A diversidade de elementos cristalográficos através dos quais as espécies aderem à superfície da arsenopirita sugere a ausência de um controle do retículo cristalino do sulfeto na distribuição do metal precioso.

\section{MORFOLOGIA DAS ESPÉCIES AURÍFERAS}

Uma proporção significativa de espécies auríferas, que ocorrem tanto em contato com a arsenopirita como de forma intersticial na matriz de quartzo-carbonato, foi examinada sob microscópio eletrônico de varredura. Mostram uma diversidade de formas 


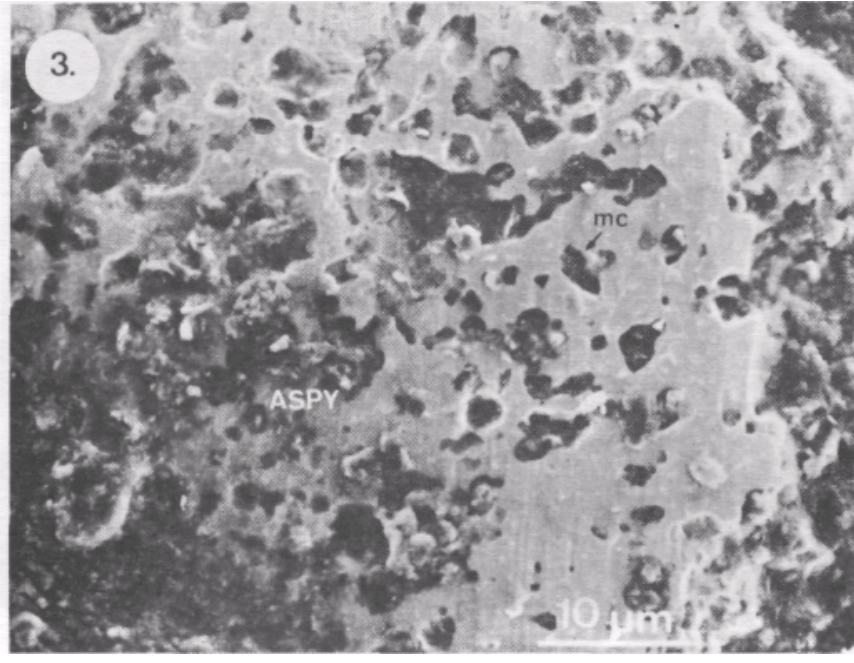

Figura 3 - Fotomicrografia obtida com microscópio eletrônico de varredura (MEV), mostrando a superficie de um grão de arsenopiríta (ASPY), em três dimensões, com abundantes microcavidades de dissolução (me)

Figure 3 - SEM photomicrography of arsenopyrite (ASPY) surfaces with abundant dissolution microcavities (MC)

que pode ser agrupada em quatro variedades texturais: 1. grãos idiomórficos, por vezes com formas octaédricas incompletas, que ocorrem achatados sobre as faces e planos de clivagem de arsenopirita (Fig. 4a); 2. grãos xenomórficos caracterizados pela sua aparência maciça em três dimensões, a qual é condicionada por fraturas na arsenopirita (Fig. 4b); 3. grãos lamelares constituídos por várias camadas (Fig. 4c), e 4. grãos compostos, nos quais se combinam formas da segunda com a terceira variedade (Fig. 4c). Os grãos lamelares e os compostos ocorrem intersticialmente entre quartzo e carbonato.

As marcas na superfície de grãos de ouro são raras e representadas nas variedades $\mathbf{l}$ e $\mathbf{2}$ por fissuras e estrias, ao passo que nas variedades $\mathbf{3}$ e $\mathbf{4}$ predominam as marcas poligonais impressas pela matriz.

DISCUSSÃO E CONCLUSÕES O entendimento dos mecanismos de deformação e de recristalização dos sulfetos tem sido auxiliado por simulações experimentais, cujos resultados incluem estrutura de deslizamento na galena, calcopirita, pirrotita e esfalerita (Graf \& Skinner 1970, Clark \& Kelly 1973, Roscoe 1975, Deb 1979). As distorções do retículo cristalino exemplificadas por macias de deformação e kink bands, em geral seguidas de evidências de recuperação expressas por subgrãos constituem uma feição comum na deformação dúctil dos sulfetos (Mookherjee 1971, Atkinson 1975), enquanto as fraturas demonstram a sua deformação rúptil. Por outro lado, a feição característica dos processos de recristalização, tanto nos metais como nos silicates, consiste no desenvolvimento de texturas poligonais, equidimensionais e isentas de deformação. Nos metais deformados, os subgrãos são produtos dos processos de recuperação, que transformam a energia interna dos cristais tensionados em trabalho, sem desenvolver novos grãos (Cotterill \& Mould 1976). Os mecanismos de deformação e de recristalização dos sulfetos são condicionados por parâmetros físico-químicos, dentre osquais se destacam temperatura, pressão, taxa de deformação, intervalo de tempo de atuação do stress e a composição do sulfeto.

No Depósito Maria Lázara, as texturas exibidas pela arsenopirita são similares àquelas produzidas pela deformação e recuperação de metais e ligas, as quais indicam que a plasticida- de intracristalina, combinadas com a cataclase, foram importantes mecanismos na deformação desse sulfeto. A presença de subgrãos demonstra que a esses mecanismos somam-se processos de recristalização não-finalizados durante a circulação de fluidos hidrotermais.

O fraturamento e a deformação plástica da estrutura cristalina da arsenopirita, somados à sua freqüente associação com os minerais auríferos, no depósito Maria Lázara, poderiam indiretamente sugerir processos de remobilização do ouro submicroscópico originalmente contido na estrutura desse sulfeto. Entretanto as espécies auríferas podem ser encontradas em níveis ricos em arsenopirita, quer seja deformada quer seja indeformada, como em níveis ricos em filossilicatos (biotita e clorita) e isentos de sulfetos. Dados químicos obtidos com auxílio de microssonda eletrônica em cristais de arsenopirita tanto fraturados como isentos de fraturas (Pulz et al. 1991b) e confirmadas com microssonda iônica (Michel et al. 1992) sugerem a inexistência de ouro submicroscópico na estrutura $\mathrm{da}$ arsenopirita. $\mathrm{O}$ ouro nativo e os bismutinetos auríferos que ocorrem nos interstícios da matriz de quartzo e carbonato mostram raras marcas poligonais impressas sobre a sua superfície, indicando que a intensa circulação de fluidos auríferos ocorreu após a recristalização da matriz. Além disso, as formas idiomórficas dos grãos de ouro aderidos à superfície da arsenopirita sugerem um estágio tardio de percolação de fluidos auríferos, posterior à intensa sulfetação do metabasalto. A preservação dessas delicadas formas dos cristais indica que a circulação tardia dos fluidos auríferos pós-data a deformação imposta pela zona de cisalhamento Carroça na região do Depósito Maria Lázara.

O processo de recristalização metamórfica de arsenopirita aurífera, permitindo a difusão total do ouro através de sua estrutura, mostra-se inapücável para a associação ouro-arsenopirita do Depósito Maria Lázara, pois as espécies auríferas ocorrem aleatoriamente sobre a superfície do sulfeto, indicando a ausência de difusão ou exsolução cristalograficamente controlada (Starling et al. 1989). Dessa forma, os dados químicos e texturais sugerem que a precipitação do metal precioso no Depósito Maria Lázara ocorreu diretamente do fluido hidrotermal, por processos que independem dos mecanismos de deformação e recristalização da arsenopirita ou da matriz.

A constante associação do ouro livre com a arsenopirita disseminada no halo de alteração do Depósito Maria Lázara pode estar relacionada com a evolução das condições físicoquímicas do fluido hidrotermal. O transporte do ouro sob condições hidrotermais é efetuado por complexos clorados (Helgeson 1969) ou por várias espécies de complexos sulfetados, incluindo $\mathrm{Au}(\mathrm{HS})_{2}, \mathrm{Au}_{2}(\mathrm{HS})_{2} \mathrm{~S}^{2-} \mathrm{e}$ ions de polissulfetos do tipo $\mathrm{S}^{2-}(\mathrm{n}>2)$ (Seward 1973, Huston \& Large 1989). A estabilidade desses complexos depende do $\mathrm{pH}$, salinidade, $\mathrm{fO}_{2}$, conteúdo de $\mathrm{H}_{2} \mathrm{~S}$, temperatura e pressão de fluido (Brown 1989, Large 1990). Qualquer variação desses parâmetros físico-químicos desestabiliza os complexos auríferos e favorece a deposição de sua carga metálica. No Depósito Maria Lázara é freqüente a ocorrência de arsenopirita com microcavidades de dissolução que constituem evidências de flutuações na $\mathrm{fS}_{2} / \mathrm{fO}_{2}$ associada aos pulsos de percolação de fluidos hidrotermais. Essas variações na $\mathrm{fS}_{2} / \mathrm{fO}_{2}$ podem ter contribuído para a deposição do ouro após a cristalização da arsenopirita.

Por outro lado, a precipitação do ouro pode ser induzida por reações eletro-químicas durante a interação do fluido hidrotermal com sulfetos preexistentes (Jean \& Bancroft 1985). As reações eletro-químicas promovem a desestabilização dos complexos auríferos devido à elevada condutividade elétrica dos sulfetos. Esta propriedade, combinada com a capacidade do sulfeto atuar como agente redutor, induz o enfraquecimento das forças de Van der Waals dos ligantes auríferos, favorecendo a precipitação do ouro nativo sobre a superfície dos sulfetos (Knipe et al 1991, Seward \& Cardile 1991). Os 



Figura 4 - Fotomicrografias de imagens tridimensionais obtidas com microscópio eletrônico de varredura (MEV), ilustrando a forma dos minerais auríferos que ocorrem no Depósito Maria Lázara, a. Grãos idiomórficos de mineral aurífero (Au) com abundantes fissuras nos seus bordos. Os grãos auriferos ocorrem aderidos às faces de arsenopirita (Aspy). b. Espécie aurífera (Au) com forma maciça condicionada pelo espaço aberto na fratura da arsenopiríta (Aspy). c. Grãos de ouro com formas lamelares $(A u L)$ e compostas (AuC). As formas compostas são definidas pela combinação de um núcleo maciço envolto por uma auréola incompleta de grãos lamelares. Reações de ataque incompletas durante a preparação da amostra preservaram relictos de quartzo $(Q z)$ e promoveram a reprecipitação da silica (Si) sobre os grãos de ouro

Figure 4 - SEM photomicrography ilustrating the forms of gold minerais in the Maria Lázara gold deposit, a. Idiomorphic gold grains (Au) with abundant fissures, adhered to the arsenopyrite faces, $\mathbf{b}$. Massive form of gold grain ( $\mathrm{Au}$ ) constrained by the open space of fracture within arsenopyrite (Aspy). c. Lamellae (Aul) and composite (AuC forms of gold grains. Composite forms are defined by a massive core within a descontinue aureole of lamellae. Incomplete reaction during sample preparation preserved quartz relics $(\mathrm{Qz})$ resilted in $(\mathrm{Si})$ reprecipitation upon gold grains

contatos planares e abruptos mostrados pela associação ouroarsenopirita no Depósito Maria Lázara demonstram que, pelo menos para o estágio tardio de percolação de fluidos auríferos, a arsenopirita serviu de substrato para a deposição do ouro.

No Depósito Maria Lázara, o sistema hidrotermal continuou em atividade após a percolação dos fluidos auríferos, como indicam as abundantes fissuras nos bordos dos grãos idiomóficos de ouro nativo. As fissuras superficiais em grãos idiomóficos sugerem processos de perda de volume (Hilmy \& Osman 1989) devido a mudanças físico-químicas no fluido hidrotermal, posteriores à deposição das espécies auríferas. Essas mudanças podem ter contribuído para a remobilização do metal precioso favorecendo, a distribuição errática dos teores de ouro no nível mineralizado.

Do exposto, conclui-se que a deposição dos minerais auríferos no Depósito Maria Lázara foi efetuada por processos independentes do metamorfismo e da deformação dos sulfetos ou da matriz hidrotermalizada. Por outro lado, as relações texturais e químicas fornecem evidências de que a deposição do ouro está relacionada com a evolução do sistema hidrotermal, sendo que a desestabilização de complexos auríferos, quer por oxidação dos fluidos, quer por reações eletroquímicas, favoreceu a precipitação do metal precioso no Depósito Maria Lázara.

Agradecimentos Os autores externam sua gratidão ao prof. E.W. Kitajima pelo apoio técnico na utilização do microscópio eletrônico de varredura do Instituto de Ciências Biológicas, Universidade de Brasília, e ao Conselho Nacional de Desenvolvimento Científico e Tecnológico $(\mathrm{CNPq})$ pelo suporte financeiro sob a forma de bolsa de doutorado (GMP) e de desenvolvimento científico regional (ESM). Ao prof. Nilson F. Botelho e aos revisores anônimos da RBG, agradecemos pelas críticas e sugestões ao texto. 


\section{REFERÊNCIAS BIBLIOGRÁFICAS}

ATKINSON, B.K. 1975. Experimental deformation of polycrystaline pyrite: effects of temperature, confining pressure, strain rate, and porosity. Econ Geol, 70:473-487.

BARKEN, B.M.; HOCHELLA, M.F., Jr.; MARSHALL, AJ ${ }^{3}$.; TURNER, A.M 1989. High resolution microscopy of gold in unoxidized ore from the Carlin mine, Nevada. Econ. Geol, 84:171-179.

BROWN, K.L. 1989. Kinetics of gold precipitation from experimental hydrothermal sulfide solutions. Econ. Geol, 6:320-327. (Monograph)

CABRI, L.J.; CHRYSSOULIS, S.L.; DE VTLLIERS, J.P.R.; LA FLAMME, J.H.G.; BUSECK, P.R. 1989. The nature of invisible gold in arsenopyrite Can. Mineral., 27:353-362.

CLARK, B.R. \& KELLY, W.C. 1973. Sulphide deformation studies: I. Experimental deformation of pyrrhotite and sphalerite to 2,000 bars and $500^{\circ} \mathrm{C}$. Econ. Geol, 68:332-352.

COTTERILL, P. \& MOULD, P.R. 1976. Recrystallization and Grain Growth in Metals. London, Surrey University Press. 409 p.

DANNI, J.C.M.; JOST, H; WINGE, M.; ANDRADE, G.F. 1986. Aspectos da evolução dos terrenos granito-greenstone: exemplo da região de Hidrolina. In: CONG. BRAS. GEOL., 34. Goiânia, 1986. Anais... Goiânia, SBG. v. 2, p. 570-584.

DEB, M. 1979. Polymetamorphism of ores in Precambrian stratiform massive sulfide deposits at Ambaji-Deri, Western India. Mineral Deposita, 14:21-31.

GRAF, J.L., Jr. \& SKINNER, BJ. 1970. Strength and deformation of pyrite and pyrrhotite. Econ. Geol, 65:206-215.

HELGESON, H.C. 1969. Thermodynamics of hydrothermal systems at elevated temperatures and pressures. Am. J. Sci., 267:729-804

HILMY, M.E. \& OSMAN, A. 1989. Remobelization of gold from a chalcopyrite-pyrite mineralization Hamash gold mine, Southeastern Desert, Egypt. Mineral Deposita, 24:244-249.

HUSTON, O.K. \& LARGE, R.R. 1989. A chemical model for the concentration of gold in volcanogenic massive sulphide deposits. Ore Geol Rev. 4(3): $171-200$

JEAN, G.E. \& BANCROFT, G.M. 1985. An XPS and SEM study of gold deposition at low temperatures on sulphide mineral surfaces: concentration of gold by adsorption/reduction. Geochim. Cosmochim. Acta, 49:979-987.

JOHAN, Z.; MARCOUX, E.; BONNEIMAISON, M. 1989. Arsenopyrite aurifere: mode de substitution de Au dans la structure de FeAsS. C.R. Acad. Sci. Fr, 308:185-191.

KNIPE, S.W.; FOSTER, R.P.; STANLEY, CJ. 1991. Hydrothermal precipitation of precious metals on sulfide substrates. In: LADEIRA, E.A. ed. Proceedings of Brazil G0W97, an International Symposium on the Geology of Gold. Belo Horizonte, 1991. Rotterdam, A.A. Balkema. p. 431-435.

LARGE, R.H. 1990. The gold-rich seafloor massive sulphide deposits of Tasmania. Geol Rundsch., 79:265-278.
MICHEL, D.; GIULIANI, G.; PULZ, G.M.; JOST, H. 1992. Evidence for multistage gold deposition in the Archean Maria Lázara gold deposit (Goiás, Brazil). Mineral Deposit Eur. J. Mineral (submetido).

MOOKHERJEE, A. 1971. Deformation of pyrite - a discussion. Econ Geol, 66:200.

NEBEL, M.; HUTCHINSON, R.W.; ZARTMAN, R. 1991. Metamorphism and polygenesis of the Madem Lakkos polymetallic sulfide deposit, Chalkidiki, Greece. Econ. Geol, 86:81-105

PICOT, P. \& MARCOUX, E. 1987. Nouvelles données sur Ia métallogenie de 1'or. C.R. Acad. Sci. Fr., 304:221-226.

PULZ, G.M. 1990. Geologia do Depósito Aurifero tipo Maria Lázara, Guarinos, Goiás. Brasilia. 132 p. (Dissertação de Mestrado, IG/UnB).

PULZ, G.M.; GIULIANI, G.; MICHEL, p.; JOST, H. 1992b. Microtexturas e composição química dos minerais metálicos do depósito de ouro Maria Lázara, Greenstone Belt Guarinos, Goiás. An. Acad. bras. Ciên., 64:169-182.

PULZ, G.M - JCST, H ; GIULIANI, G ; MICHEL, D. 1991a. Maria Lázara gold deposit (Goiás state, Brazil): an example of intense fluid/rock interaction associated with a triple point structure. In: PAGEL, M. \& LEROY, J.L. eds. Source, Transport and Deposition of Metals. Rotterdam, A.A. Balkema. p. 117-118.

PULZ, G.M · JOST, H. GIULIANI, G.; MICHEL, D. 1992a. Evidências mineralógicas e estruturais da percolação episódica de fluidos hidrotermais no depósito aurífero Maria Lázara (Goiás). An. Acad. bras. Ciên. (no prelo).

PULZ, G.M · MICHEL, D · GIULIANI, G · JOST, H. 1991b. Zonação química de arsenopiritas: um indicador de múltiplos episódios de mineralização em zonas de cisalhamento. In: CONG. BRAS. GEOQUÍMICA, 3 - CONG. DE GEOQUÍMICA DOS PAIISES DE LÍNGUA PORTUGUESA, 1. São Paulo, 1991. Resumos... São Paulo, SBGq. v. 2, p. 676-679.

ROSCOE, WE. 1975. Experimental deformation of natural chalcopyrite at temperatures up to $300^{\circ} \mathrm{C}$ over the strain rate $10^{\prime \prime 2}$ to $10^{\prime \prime *}$ sec $^{\prime 1}$. Econ. Geol., 70:454-472.

SEWARD, T.M. 1973. This complexes of gold in hydrothermal ore solutions. Geochim Cosmochim. Acta 37:379-399.

SEWARD, T.M \& CARDILE, C.M. 1991. Gold adsorption onto colloidal sulphide substrates. In: PAGEL, M. \& LEROY, J.L. eds. Source, Transport and Deposition of Metals. Rotterdam, A. A. Balkema. p. 707-708.

STARLING, A.; GILLIGAN, J.M.; CARTER, A.H.C.; FOSTER, R.P.; SAUNDERS, R.A. 1989. High-temperature hydrothermal precipitation of precious metals on the surface of pyrite. Nature, 340:298-300.

YOKES, F.M. 1969. A review of the metamorphism of sulphide deposits. Earth-Sci. Rev., 5:99-143.

MANUSCRITO A730

Recebido em 20 de julho de 1992 Revisão do autor em 19 de agosto de 1992 Revisão aceita em 19 de agosto de 1992 